\title{
Effects of laser therapy versus laser acupuncture on rheumatoid arthritis elderly patients
}

\author{
Running Title \\ Laser therapy versus laser acupuncture on rheumatoid arthritis elderly \\ Short Title \\ Laser therapy vs puncture on RA elderly \\ Afnan Sedky Adly ${ }^{1,2 *}$, Zahra M. Serry ${ }^{3}$, Mohamed Shehata ${ }^{4}$, Mohamed Amr ${ }^{5}$, \\ Heba Ahmed Ali Abdeen ${ }^{3}$ \\ ${ }^{1}$ Faculty of Physical Therapy, Cardiovascular-Respiratory Disorders and Geriatrics, Cairo \\ University, Cairo, Egypt \\ ${ }^{2}$ Faculty of Physical Therapy, Internal Medicine, Beni-Suef University, Beni-Suef, Egypt \\ ${ }^{3}$ Department of Cardiopulmonary Disorder and Geriatrics, Faculty of Physical Therapy, \\ Cairo University, Cairo, Egypt. \\ ${ }^{4}$ Department of Clinical Pathology, Faculty of Medicine, Cairo University, Cairo, Egypt. \\ ${ }^{5}$ National Institute of Laser Enhanced Sciences, Cairo University, Cairo, Egypt. \\ *Corresponding author: Afnan Sedky Adly \\ Email: afnansedky@yahoo.com \\ Mobile: +20 01145559778 \\ Address: Cairo University Road, Cairo University, Egypt.
}

\section{Abstract}

Objective

Rheumatoid arthritis (RA) is regarded as the commonest and one of most known inflammatory arthritis. Our study was to determine the effects of laser therapy and laser acupuncture therapy on $\mathbf{R A}$ elderly patients.

Subjects and Methods

Forty patients with age of 65 to 75 years were being randomly allocated to 2 groups. Group(A) included twenty participants having $R A$ treated via low level laser therapy. Group(B) included twenty participants having $R A$ treated via laser acupuncture. All the patients underwent treatment by methotrexate. Treatments were provided three times each week to the two groups for twelve weeks.Independent laboratory technician and statistician were blinded to the study groups.

Results

There was significant reduction in scores of RAQoL questionnaire pre as well as post treatments for both the groups, with $p<0.05$. For HAQ questionnaire, significant difference was in the group treated with laser acupuncture. The IL-6 inflammatory marker had a significant reduction in pre as well as post treatments in only the group treated with laser 
acupuncture, with $p<0.05$. In the MDA marker, it had a significant reduction in both groups for pre as well as post treatments. There was also a significant increase to the ATP antioxidant marker in pre as well as post treatments for both the groups, $p<0.05$.

Conclusions

This study suggested that the laser acupuncture can offer promising therapeutic benefits to assess in rheumatoid arthritis treatments; those therapeutic effects might be from inflammation reduction and oxidation stress alleviation, in addition to improvements in the antioxidants and energy status for $R A$ patients.

Keywords: puncture; laser acupuncture; rheumatoid arthritis; elderly.

\section{Introduction}

The disorder of rheumatoid arthritis (RA) is considered from the systemic chronic arthritis which is immune mediated and can affect mainly synovial joints, probably with symmetrical patterns. It is one of the chronic disabling conditions that probably cause deformity and pain [1,2].

Current evidences showed that the early investigations and prompt diagnosis of the definitive disease-modifying treatments could avoid or delay progressions in addition to enabling patients to retain functions, which will otherwise be lost during this frequently disabling and progressive disease. The RA is estimated for affecting between around 0.5 and $2 \%$ of populations all over the world [3].

Generally speaking, this disorder is being similarly common all over the world, even though there was evidences of variations along inferior prevalence rates of the Southern Europe when compared to North America and the Northern Europe [4].

In addition, there might be some reduction in rates of the developing countries when compared to developed world, findings which have been related to the environmental influences of the urbanization, even though the exact causes are not identified [5].

The native health assessment questionnaire (HAQ) is still considered as one of the gold standards for assessing the functional status of RA patients. This questionnaire is widely validated in several cultural settings, it is very sensitive to changes. It also has been extensively utilized in the clinical trials for assessment of physical function. [6]

Rheumatoid arthritis quality of life index (RAQoL) is a disorder specific tool related only to patients having Rheumatoid Arthritis. The index is considered self-explanatory and need no specific training or education for the patients.[7]

Several researches have reported some trends for reduction in rate of recurrence, more precisely in countries having high rates of diseases even though such trends are far from certain. Nevertheless, the few number of researches for most the world areas as well as lacking of incidence researches for the developing countries suggests that global epidemiology knowledge 
of the RA is still limited $[8,9]$. Our study was done to determine the effects of both laser therapy and laser puncture therapy on RA elderly patients.

\section{Subjects and methods}

Forty patients from both sexes with age of 65 to 75 years, who were selected of the outpatient clinics, in Cairo University. Those patients were allocated randomly and equally into 2 groups; In group (A): 20 participants with rheumatoid arthritis treated by low level laser therapy. Group (B): 20 participants with rheumatoid arthritis treated by the laser puncture therapy. All the subjects received treatments with a disease-modifying antirheumatic drug (i.e. methotrexate). Treatments were given 3 times during a week for 12 weeks to the two groups.

A research ethical approval has been obtained before this study start. The aim, potential risks and nature of this study were clarified to every patient and the written informed consents were signed prior to participations.

\section{Inclusion criteria}

Inclusion of patients depended on meeting the following:

- They were from 65 to 75 years.

- Both sexes included.

- All patients referred from physician (immunologist) diagnosed with rheumatoid arthritis.

\section{Exclusion criteria}

All patients were subjected to complete clinical examinations and complete clinical history for exclusions of the following:

- Patients with a disease that overlap with any connective tissue disorder, like the systematic lupus erythromatosus \& systematic sclerosis.

- Patients with diabetes mellitus.

- Cognitive or psychiatric problems.

- History of malignancies.

- History of steroidal injections (within 3 months).

- Patients with hemorrhagic tendencies.

- Smokers.

- Alcoholics.

\section{Measuring Tools:}

- Kits and Tubes of blood samples for the analysis of:

- The antioxidant markers: concentrations of plasma ATP.

- The oxidative markers: malondialdehyde in plasma (MDA).

- The inflammatory markers: interleukin-6 in plasma (IL-6).

- The Health Assessment Questionnaire (HAQ).

- The rheumatoid Arthritis Quality of Life (RAQoL) index. 
All the blood samples RAQoL questionnaire were collected and analyzed by an independent laboratory technician and a statistician who were blinded to the groups of the study.

\section{Treatment Tools:}

Low intensity laser probe device: The GaAlAs lasers system with 904nm, and class 3B wavelength.

\section{Procedures for treatment: \\ Preparatory procedures}

Each participant was informed about the significance of study as well as the experimental process. Participant's skin was cleaned by alcohol in order to decrease skin resistance and eliminate skin debris. Affording noiseless space and privacy for working before the initial treatments.

\section{Treatment procedures}

The study group (A) received low level laser therapy at trigger points, the study group (B) received laser puncture.

Group (A): Patients in this group were subjected to low intensity laser probe device: the GaAlAs lasers system with $904 \mathrm{~nm}$, and beam area 0.785 , and class 3B wavelength, C/W, 500 $\mathrm{mW}$.

The laser treatment targets three trigger points in each affected joint. The incident power density per point is approximately $650 \mathrm{~mW} / \mathrm{cm} 2$, delivering an energy density per point per irradiation of $20.1 \mathrm{~J} / \mathrm{cm} 2$, irradiation time $30 \mathrm{sec}$.

Group (B): The patients in this group were subjected to laser puncture: Low intensity laser probe equipment, Gallium Aluminum Arsenide laser device with $904 \mathrm{~nm}$, class of 3B, the beam area equal to $1 \mathrm{~cm} 2$, mode of pulsed emission, power density equal to $100 \mathrm{~mW} / \mathrm{cm} 2$, power equal to $100 \mathrm{~mW}$, energy density equal to $4 \mathrm{~J} / \mathrm{cm} 2$, energy dosage equal to $4 \mathrm{~J}$, frequency $100,000 \mathrm{~Hz}$, irradiation time equal to $40 \mathrm{sec}$, probe held fixed on the skin by $90^{\circ}$ angle, along minor pressure.Puncture points that have been used were liver 3 (LR3), the stomach 25,36 (ST25\& ST36), the small intestine 3,4 (SI3\&SI4), the large intestine 4,11 (LI4\&LI11), the spleen 6,9 (SP6\&SP9), the gall bladder 25,24 (GB25\&GB34), the heart 7 (HT7).

Each puncture point is identified by the meridian on which it is located and its number in the point sequence on that channel. The points selectedwere found to have anti-inflammatory and anti-oxidative systemic effects as been reported in many studies.[10, 11]

Statistical analysis of data was done using independent t-test for comparison of the 2 groups. Means and standard deviations were also calculated. The significance level was set at $\mathrm{p}<0.05$. 


\section{Results}

Table 1 demonstrate the mean and the standard deviation of the patients with respect to the age of both of the groups.

Table 1: Mean as well as Standard Deviation for ages of patients by groups.

\begin{tabular}{|l|c|c|}
\hline Group & Age mean & $\begin{array}{c}\text { Age standard } \\
\text { deviation }\end{array}$ \\
\hline \hline Group A $(\mathrm{n}=20)$ & 70.95 & 3.53 \\
\hline Group B $(\mathrm{n}=20)$ & 70.3 & 3.89 \\
\hline \hline Total & 70.62 & 3.68 \\
\hline
\end{tabular}

Statistical analysis of data by using an independent t-tests for the 2 groups for evaluating the changes in Rheumatoid Arthritis Qualities of Life (RAQoL) scale, Health Assessments questionnaires (HAQ), interleukin-6 in plasma (IL-6), malondialdehyde in plasma (MDA), adenosine tri-phosphate (ATP) concentrations in plasma as presented in table 2.

Table 2: The comparison analysis of RAQoL, HAQ, IL6, MDA and ATP average between group A \& group B, pre \& post at entry of the study

\begin{tabular}{|c|c|c|c|c|c|}
\hline $\begin{array}{c}\text { Outcom } \\
\text { e }\end{array}$ & Items & $\begin{array}{c}\text { Group A } \\
\text { Mean } \pm \text { SD }\end{array}$ & $\begin{array}{c}\text { Group B } \\
\text { Mean } \pm \text { SD }\end{array}$ & t-value & Significance \\
\hline \multirow[t]{2}{*}{ RAQoL } & $\begin{array}{l}\text { Pre- } \\
\text { treatment }\end{array}$ & $17.75 \pm 5.65$ & $15.5 \pm 6.5$ & 1.168 & NS \\
\hline & $\begin{array}{l}\text { Post- } \\
\text { treatment }\end{array}$ & $14.9 \pm 5.24$ & $10.85 \pm 6.25$ & 2.220 & $\mathbf{S}$ \\
\hline \multirow[t]{2}{*}{ HAQ } & $\begin{array}{l}\text { Pre- } \\
\text { treatment }\end{array}$ & $1.23 \pm 0.39$ & $1.41 \pm 0.41$ & 1.419 & NS \\
\hline & $\begin{array}{l}\text { Post- } \\
\text { treatment }\end{array}$ & $1.16 \pm 0.4$ & $1.15 \pm 0.38$ & 0.0201 & NS \\
\hline \multirow[t]{2}{*}{ IL6 } & $\begin{array}{l}\text { Pre- } \\
\text { treatment }\end{array}$ & $106.33 \pm 63.76$ & $98 \pm 67.6$ & 0.4009 & NS \\
\hline & $\begin{array}{l}\text { Post- } \\
\text { treatment }\end{array}$ & $104.675 \pm 64.71$ & $68.64 \pm 53.35$ & 1.921 & NS \\
\hline \multirow[t]{2}{*}{ MDA } & $\begin{array}{l}\text { Pre- } \\
\text { treatment }\end{array}$ & $8.65 \pm 0.51$ & $8.47 \pm 0.36$ & 1.280 & NS \\
\hline & $\begin{array}{l}\text { Post- } \\
\text { treatment }\end{array}$ & $8.24 \pm 0.57$ & $4.88 \pm 0.31$ & 22.93 & $\mathbf{S}$ \\
\hline
\end{tabular}


European Journal of Molecular \& Clinical Medicine

ISSN 2515-8260 Volume 08, Issue 03, 2021

\begin{tabular}{|l|l|c|c|c|c|}
\hline ATP & $\begin{array}{l}\text { Pre- } \\
\text { treatment }\end{array}$ & $18.75 \pm 2.3$ & $19.8 \pm 1.07$ & 1.853 & NS \\
\cline { 2 - 5 } & $\begin{array}{l}\text { Post- } \\
\text { treatment }\end{array}$ & $33.3 \pm 11.29$ & $66.24 \pm 2.63$ & 12.7 & S \\
\hline
\end{tabular}

\section{Discussion}

The present study showed that laser puncture had more significant improvement in IL-6, CRP, MDA and ATP concentrations in patients with RA when compared with laser therapy.

Similarly, Atifet, al., 2016 studied anti-inflammatory and antioxidant effects of the laser puncture in thirty patients having RA. The first group showed significant increase on the plasma superoxide dismutase (SOD), catalase activities, glutathione reductase (GR), plasma ATP concentrations, and blood glutathione (GSH), in comparison with those prior to treatment $(\mathrm{P}<0.005)$. Furthermore, the results showed significant reduction in the Malondyaldehyde, $\mathrm{C}$ reactive protein, nitrite and nitrate, interleukins levels and significant reduction in erythrocyte sedimentation rate (ESR) \& glutathione peroxidase (GPx) activity in the laser treated patients, in comparison to those before therapy (P less than 0.005). The patients with RA treated by the laser puncture revealed very significant decrease in disorder activity ( $\mathrm{P}$ less than 0.005 ) based on the DAS28 scores. [12]

In this study, increasedlevels ofthe malondyaldehyde was perceived in the serum of the rheumatoid arthritis patients. Those results were on agreementwith prior findings thatinformed great levels of malondyaldehyde on serum as well as RA patients' synovial fluids. [13, 14]

Synovial fluid of rheumatoid arthritis elderly patients contained elevated levels of malondyaldehyde, suggest in vivo elevated lipids peroxidation. This end product of lipid peroxidation would be transmitted from joints synovial fluid toward the circulation, elevating the malondyaldehyde levels, as being detected on this study. Thus, increased free radicals generation on inflamed joints as well as impaired antioxidant systems were implicated on rheumatoid arthritis. Laser puncture has been effective on reduction of the MDA plasma level, which indicate its high antioxidant activities. Furthermore, that therapeutic laser modality has also been effective on improvement of the levels of the antioxidant markers (ATP) for patients with RA. Both of those effects indicated the great potential of the laser therapy on suppression of the oxidative stress, consequently, on reduction of the oxidative tissues damage.

Previous studies on RA have revealed expressions of the cytokines and the other inflammation markers on elderly patients having rheumatoid arthritis is mainly in charge of the continuous destruction of the articular bone and cartilage. $[15,16]$

In the rheumatoid synovium, the interleukin-6 was considered the greatest plentifully found cytokine. As presented in this investigation, all of the investigated patients with RA were found $+v e$ for the interleukin- 6 and this can be taken as an indication of the significant role of that 
interleukin on the RA pathogenesis. The self-antigens-activated Tcells\& macrophages for stimulating immune response throughout inflammations secrete the interleukin- 6 and are stimulating both the autoimmune and inflammatory process in the rheumatoid arthritis. [17]

Recent evidence found that interleukin-6 has a main role in the pain mechanisms in RA. The translational far-reaching effects of the interleukin- 6 in RA are starting to be valued in recent studies. These effects were also supported by the ubiquitous expressions of interleukin- 6 transsignaling mechanisms. It was found that interleukin- 6 can mediate the pain by direct acting on nociceptive systems thereby negatively impacting quality of life. [18]

Laser treatment was more effective on reduction of the levels of interleukin-6 plasma in the rheumatoid arthritis patients when compared with laser puncture, which indicate its great antiinflammatory activities on RA. Those results indicated that laser treatment may have suppressive actions on inflammation mediators.

The activity of disorder in RA patients in this study was evaluated utilizing the RAQoL scale and HAQ questionnaires. The outcomes showed a great score for the disorder activity, conveyed in RAQoL for the 2 groups prior to therapy. In addition, the outcomes shown a major decrease in the disorder activity scores conveyed in RAQoL for the 2 groups after treatment. Therefore the improvement found in this study can be attributed to the possible systemic effects of laser use. Although much of the source of inflammatory pain (which is the major factor that can affect disease activity of RA patients) can be peripherally (i.e., joints capsules, muscles and skin, ligaments), the central nervous system was found to be responsible for interpretation and perception of the pain that occurs, and mainly long-term pain of RA [19]. This has made the systemic therapeutic effects of laser ideal for pain reduction.

\section{Limitation}

The sample size is not considerably large, which may affect this study reliability of the results since it is possible to cause variability that could lead to biases.

\section{Conclusion}

Laser puncture can improve significantly several markers including HAQ, and IL-6 in the elderly patients having rheumatoid arthritis. Laser puncture offers greatest therapeutic benefits for treating rheumatoid arthritis; these satisfying effects may be due to alleviation of oxidative stress, reduction of inflammation, and improvement of the energetic status in elderly with RA.

\section{References}

[1] Burmester GR, Pope JE. Novel treatment strategies in rheumatoid arthritis. The Lancet. 2017;389:2338-48.

[2] Padjen I, Crnogaj MR, Anić B. Conventional disease-modifying agents in rheumatoid arthritis-a review of their current use and role in treatment algorithms. Reumatologia. 2020;58:390. 


\section{European Journal of Molecular \& Clinical Medicine}

\section{ISSN 2515-8260 Volume 08, Issue 03, 2021}

[3] Almutairi KB, Nossent JC, Preen DB, Keen HI, Inderjeeth CA. The prevalence of Rheumatoid Arthritis: A systematic review of population-based studies. The Journal of rheumatology. 2021;48:669-76.

[4] Abhishek A, Doherty M, Kuo C-F, Mallen CD, Zhang W, Grainge MJ. Rheumatoid arthritis is getting less frequent - results of a nationwide population-based cohort study. Rheumatology. 2017;56:736-44.

[5] Otón T, Carmona L. The epidemiology of established rheumatoid arthritis. Best Practice \& Research Clinical Rheumatology. 2020:101477.

[6] Pincus T, Keysor J, Sokka T, Krishnan E, Callahan LF. Patient questionnaires and formal education level as prospective predictors of mortality over 10 years in $97 \%$ of 1416 patients with rheumatoid arthritis from 15 United States private practices. The Journal of rheumatology. 2004;31:229-34.

[7] Garrow AP, Papageorgiou AC, Silman AJ, Thomas E, Jayson MI, Macfarlane GJ. Development and validation of a questionnaire to assess disabling foot pain. Pain. 2000;85:10713.

[8] Safiri S, Kolahi AA, Hoy D, Smith E, Bettampadi D, Mansournia MA, et al. Global, regional and national burden of rheumatoid arthritis 1990-2017: a systematic analysis of the Global Burden of Disease study 2017. Annals of the rheumatic diseases. 2019;78:1463-71.

[9] Fazal SA, Khan M, Nishi SE, Alam F, Zarin N, Bari MT, et al. A clinical update and global economic burden of rheumatoid arthritis. Endocrine, Metabolic \& Immune Disorders-Drug Targets (Formerly Current Drug Targets-Immune, Endocrine \& Metabolic Disorders). 2018;18:98-109.

[10] Lee M, Shin B-C, Ernst E. Acupuncture for rheumatoid arthritis: a systematic review. Rheumatology. 2008;47:1747-53.

[11] Kim D, Bae C-H, Jun YL, Jeon H, Koo S, Kim S. Acupuncture alters pro-inflammatory cytokines in the plasma of maternally separated rat pups. Chinese journal of integrative medicine. 2017;23:943-7.

[12] Attia AM, Ibrahim FA, Abd El-Latif NA, Aziz SW, Elwan AM, Abdel Aziz AAA, et al. Therapeutic antioxidant and anti-inflammatory effects of laser acupuncture on patients with rheumatoid arthritis. Lasers in Surgery and Medicine. 2016;48:490-7.

[13] Grönwall C, Amara K, Hardt U, Krishnamurthy A, Steen J, Engström M, et al. Autoreactivity to malondialdehyde-modifications in rheumatoid arthritis is linked to disease activity and synovial pathogenesis. Journal of autoimmunity. 2017;84:29-45.

[14] Mikuls TR, Duryee MJ, Rahman R, Anderson DR, Sayles HR, Hollins A, et al. Enrichment of malondialdehyde-acetaldehyde antibody in the rheumatoid arthritis joint. Rheumatology. 2017;56:1794-803.

[15] Nerurkar L, Siebert S, McInnes IB, Cavanagh J. Rheumatoid arthritis and depression: an inflammatory perspective. The Lancet Psychiatry. 2019;6:164-73.

[16] Altobelli E, Angeletti PM, Piccolo D, De Angelis R. Synovial fluid and serum concentrations of inflammatory markers in rheumatoid arthritis, psoriatic arthritis and osteoarthitis: a systematic review. Current rheumatology reviews. 2017;13:170-9. 
[17] Schinnerling K, Aguillón J, Catalán D, Soto L. The role of interleukin-6 signalling and its therapeutic blockage in skewing the $\mathrm{T}$ cell balance in rheumatoid arthritis. Clinical \& experimental immunology. 2017;189:12-20.

[18] Choy EH, Calabrese LH. Neuroendocrine and neurophysiological effects of interleukin 6 in rheumatoid arthritis. Rheumatology. 2018;57:1885-95.

[19] McWilliams DF, Walsh DA. Pain mechanisms in rheumatoid arthritis. Clin Exp Rheumatol. 2017;35:94-101. 